\title{
A variant of McDougall-Wotherspoon method for finding simple roots of nonlinear equations
}

\author{
Nataša Glišović, Nebojša M. Ralević, Dejan Ćebić
}

\begin{abstract}
Recently, McDougall and Wotherspoon have developed a simple modification to the standard Newton method for finding simple roots of nonlinear equations. Their method is based on the arithmetic mean and achieves approximately 2.4 convergence order. This research presents a new iteration scheme based on the harmonic mean with the same convergence order and the comparison with the original McDougall-Wotherspoon method. Several test examples have been used and the results show good numerical behavior of the new method.

Keywords: McDougall and Wotherspoon method, root-finding, iterative method, numerical method.
\end{abstract}

\section{Introduction}

One of the most frequent problems in applied mathematics is finding the roots of nonlinear equation $f(x)=0$. For a vast number of real-life applications, the exact roots cannot be found, and therefore some iterative methods are being employed to determine the approximate roots with certain level of accuracy. When a sequence $\left\{x_{n}\right\}$ generated by an iterative process converges to a exact root $\alpha$ such that the following equation holds

$$
\lim _{x \rightarrow \infty} \frac{\left|x_{n+1}-\alpha\right|}{\left|x_{n}-\alpha\right|^{p}}=C
$$

for some constant $C \neq 0$, and $p \geq 1$, than it can be said that the iterative method has the convergence order $p$, and $C$ is asymptotic error constant.

Probably the best-known root-finding iterative method is Newton's method with the form

$$
x_{n+1}=x_{n}-\frac{f\left(x_{n}\right)}{f^{\prime}\left(x_{n}\right)}, n=0,1,2, \ldots
$$

Manuscript received January 27 2018; accepted May 25, 2018.

N. Glišović is with the State University of Novi Pazar, Novi Pazar, Serbia; M. Ralević and D. Ćebić are with the University of Novi Sad, Faculty of Technical Sciences, Novi Sad, Serbia. 
If initial approximation $x_{0}$ is chosen sufficiently close to the simple root $\alpha$ (which means that $f(\alpha)=0$ and $f^{\prime}(\alpha) \neq 0$ ), Newton's method has quadratic convergence.

Based on this method, many researchers have developed various modifications and multistep iteration schemes with improved convergence order. As a measure of efficiency, in 1960. Ostrowski [8] introduced the coefficient $I=p^{1 / q}$, where $p$ is the convergence order, and $q$ is the number of function of derivative evaluations per iteration. Therefore, since Newton's method (1) reaches convergence order two with one function and one first derivative evaluation per iteration, its efficiency index is $2^{1 / 2} \approx 1.4142$. Higher convergence order or efficiency usually demand additional function/derivative evaluations. For example, Weerakoon and Fernando [11] proposed two-step method

$$
\begin{aligned}
y_{n} & =x_{n}-\frac{f\left(x_{n}\right)}{f^{\prime}\left(x_{n}\right)}, \\
x_{n+1} & =x_{n}-\frac{f\left(x_{n}\right)}{\frac{1}{2}\left(f^{\prime}\left(x_{n}\right)+f^{\prime}\left(y_{n}\right)\right)},
\end{aligned}
$$

using one function evaluation and the arithmetic mean of two derivative evaluations. Using the harmonic mean in the denominator of method (2) instead of the arithmetic mean, Özban developed a similar third-order root-finding method [9].

Beside those methods, for the purpose of comparison with the new method, some Newton-type methods are presented as follows:

- Frontini-Sormani method [2]

$$
x_{n+1}=x_{n}-\frac{f\left(x_{n}\right)}{f^{\prime}\left(x_{n}-f\left(x_{n}\right) /\left(2 f^{\prime}\left(x_{n}\right)\right)\right)},
$$

- Kou-Li-Wang method [5]

$$
x_{n+1}=x_{n}-\frac{f\left(x_{n}+f\left(x_{n}\right) / f^{\prime}\left(x_{n}\right)\right)-f\left(x_{n}\right)}{f^{\prime}\left(x_{n}\right)},
$$

- Wang method [10]

$$
x_{n+1}=x_{n}-\frac{4 f\left(x_{n}\right)}{f^{\prime}\left(x_{n}\right)+3 f^{\prime}\left(x_{n}-2 f\left(x_{n}\right) /\left(3 f^{\prime}\left(x_{n}\right)\right)\right)} .
$$

All presented third-order method have efficiency index $3^{1 / 3} \approx 1.4422$.

In contrast to the displayed methods, there are some other root-finding techniques which reuse information from the previous iterations in order to improve convergence speed. The methods of this type are called methods with memory. The best-known method with memory is the secant method, which can be also viewed as a modification of Newton's 
method (1), whereas the first derivative $f^{\prime}\left(x_{n}\right)$ is approximated by the divided difference $\left(f\left(x_{n}\right)-f\left(x_{n-1}\right)\right) /\left(x_{n}-x_{n-1}\right)$. Although its efficiency index is $0.5(1+\sqrt{5}) \approx 1.618$ which is larger than method (1), the secant method has certain drawbacks described in [7] with more details.

\section{McDougall-Wotherspoon method}

Motivated by studies related to the algorithms for seawater analysis [3, 6], McDougall and Wotherspoon have derived a multistep iterative method with memory [7], as a new modification of the classical Newton's method, given by

$$
\begin{aligned}
& \hat{x}_{0}=x_{0}, \\
& x_{1}=x_{0}-\frac{f\left(x_{0}\right)}{f^{\prime}\left(\frac{1}{2}\left(x_{0}+\hat{x}_{0}\right)\right)}=x_{0}-\frac{f\left(x_{0}\right)}{f^{\prime}\left(x_{0}\right)},
\end{aligned}
$$

followed by

$$
\begin{aligned}
\hat{x}_{n} & =x_{n}-\frac{f\left(x_{n}\right)}{f^{\prime}\left(\frac{1}{2}\left(x_{n-1}+\hat{x}_{n-1}\right)\right)}, \\
x_{n+1} & =x_{n}-\frac{f\left(x_{n}\right)}{f^{\prime}\left(\frac{1}{2}\left(x_{n}+\hat{x}_{n}\right)\right)},
\end{aligned}
$$

for $n \geq 1$. For example, the approximation $x_{2}$ is calculated using $f\left(x_{1}\right)$ and the derivative evaluated at the arithmetic mean of $x_{1}$ and $\hat{x}_{1}$. The same value of that derivative is reused in the next step to get $x_{3}$.

McDougall and Wotherspoon have shown that method (6) achieves the convergence order $1+\sqrt{2} \approx 2.4142$, and consequently the efficiency index 1.5538 (for the initial approximation $x_{0}$ chosen close to the root $\alpha$ ). Furthermore, they have verified its good numerical behavior compared to existing methods through several test examples.

\section{A new method and the convergence analysis}

If the arithmetic mean of $x_{n}$ and $\hat{x}_{n}$ is replaced by its harmonic mean, preserving $\hat{x}_{0}$ and $x_{1}$, for $n \geq 1$ the new iterative scheme can be obtained

$$
\begin{aligned}
\hat{x}_{n} & =x_{n}-\frac{f\left(x_{n}\right)}{f^{\prime}\left(\frac{2 x_{n-1} \hat{x}_{n-1}}{x_{n-1}+\hat{x}_{n-1}}\right)}, \\
x_{n+1} & =x_{n}-\frac{f\left(x_{n}\right)}{f^{\prime}\left(\frac{2 x_{n} \hat{x}_{n}}{x_{n}+\hat{x}_{n}}\right)} .
\end{aligned}
$$


From Taylor's expansion about $\alpha$, we have

$$
f(x)=f^{\prime}(\alpha) \cdot e \cdot\left(1+c_{2} e+c_{3} e^{2}+c_{4} e^{3}+\ldots\right),
$$

and

$$
f^{\prime}(x)=f^{\prime}(\alpha) \cdot\left(1+2 c_{2} e+3 c_{3} e^{2}+4 c_{4} e^{3}+\ldots\right),
$$

where $e=x-\alpha$ and $c_{i}=(1 / i !) f^{(i)}(\alpha) / f^{\prime}(\alpha)$, for $i=2,3, \ldots$ Let the errors $e_{n}$ and $\hat{e}_{n}$ be defined as $x_{n}-\alpha$ and $\hat{x}_{n}-\alpha$, respectively. Thus, from Taylor's expansion of the harmonic mean of $x_{n}$ and $\hat{x}_{n}$ about $(\alpha, \alpha)$, it is easy to get the following approximation

$$
\frac{2 x_{n} \hat{x}_{n}}{x_{n}+\hat{x}_{n}} \approx \alpha+\frac{1}{2} e_{n}+\frac{1}{2} \hat{e}_{n} .
$$

Therefore, repeated substitutions of (8), (9) and (10) into the consecutive steps of method (7) yield to the following approximations of errors:

$$
\begin{aligned}
& \hat{e}_{0} \sim e_{0}, \hat{e}_{1} \sim 2 c_{2}^{2} e_{0}^{3}, \hat{e}_{2} \sim 2 c_{2}^{6} e_{0}^{7}, \hat{e}_{3} \sim 8 c_{2}^{16} e_{0}^{17}, \ldots \\
& e_{1} \sim c_{2} e_{0}^{2}, \quad e_{2} \sim 2 c_{2}^{4} e_{0}^{5}, \quad e_{3} \sim 4 c_{2}^{11} e_{0}^{12}, e_{4} \sim 32 c_{2}^{28} e_{0}^{29}, \ldots
\end{aligned}
$$

Hence, the sequence of powers of $e_{0}$ for errors $e_{i}(i \geq 1)$ taken after full iterations gives values $2,5,12,29,70,169,408, \ldots$ which is the same as the result generated by McDougallWotherspoon method (6). Let $P_{i}$ be the $i$-th term of that sequence, than for $i \geq 2, P_{i}$ can be calculated by

$$
P_{i}=2 P_{i-1}+P_{i-2} \text {. }
$$

Dividing (11) by $P_{i-1}$ and taking the limit, we get the equation $p=2+1 / p$, where $p=$ $\lim _{j \rightarrow \infty}\left(P_{j} / P_{j-1}\right), j \in \mathbf{N}$. Its positive solution $p=1+\sqrt{2} \approx 2.4142$ represents the convergence order of the method (7).

\section{Numerical comparison}

From the above discussion it is clear that methods (6) and (7) have the same theoretical properties such as the convergence order and the efficiency index. For their numerical comparison, the following test examples taken from $[1,7]$ have been employed

$$
\begin{aligned}
& f_{1}(x)=x^{2}-e^{x}-3 x+2, x_{0}=3, \alpha=0.257302854 \ldots \\
& f_{2}(x)=x e^{x^{2}}-\sin ^{2} x+3 \cos x+5, \quad x_{0}=-2, \alpha=-1.207647827 \ldots \\
& f_{3}(x)=e^{x^{2}+7 x-30}-1, x_{0}=3.25, \quad \alpha=3 \\
& f_{4}(x)=\ln \left(x^{2}+x+2\right)-x+1, \quad x_{0}=3, \alpha=4.152590736 \ldots
\end{aligned}
$$

Tables 1-4 display the numerical results organized in the last four columns. The first column presents the methods used for calculations. Newton's method (1), the Weerakoon-Fernando 
method (2), Özban's variant of method (2), the Frontini-Sormani method (3), the Kou-LiWang method (4), Wang's method (5) and the McDougall-Wotherspoon method (6) are denoted by "NM", "WFM", "OM", "FSM", "KLWM", "WM" and "McDWM", respectively, while the new iterative scheme (7) is denoted by "NewM".

Table 1 Numerical results for $f_{1}(x)$

\begin{tabular}{lllll}
\hline method & it & $\mathrm{nFe}$ & $\left|f\left(x_{i}\right)\right|$ & $r_{c}$ \\
\hline NM & 8 & 16 & $2.28 \cdot 10^{-25}$ & 2.0000 \\
WFM & 6 & 18 & $2.80 \cdot 10^{-16}$ & 3.0000 \\
OM & 6 & 18 & $1.33 \cdot 10^{-22}$ & 3.0000 \\
FSM & 6 & 18 & $4.85 \cdot 10^{-25}$ & 3.0000 \\
KLWM & 6 & 18 & $5.65 \cdot 10^{-13}$ & 3.0000 \\
WM & 5 & 15 & $1.71 \cdot 10^{-33}$ & 3.0000 \\
McDWM & 7 & 14 & $5.88 \cdot 10^{-50}$ & 2.4147 \\
NewM & 7 & 14 & $8.97 \cdot 10^{-55}$ & 2.4148 \\
\hline
\end{tabular}

Table 2 Numerical results for $f_{2}(x)$

\begin{tabular}{lllll}
\hline method & it & $\mathrm{nFe}$ & $\left|f\left(x_{i}\right)\right|$ & $r_{c}$ \\
\hline NM & 11 & 22 & $1.08 \cdot 10^{-4}$ & 2.0000 \\
WFM & 7 & 21 & $1.76 \cdot 10^{-4}$ & 3.0000 \\
OM & 7 & 21 & $5.99 \cdot 10^{-10}$ & 3.0000 \\
FSM & 7 & 21 & $4.66 \cdot 10^{-7}$ & 3.0000 \\
KLWM & 7 & 21 & $2.44 \cdot 10^{-10}$ & 3.0000 \\
WM & 7 & 21 & $6.22 \cdot 10^{-6}$ & 3.0000 \\
McDWM & 9 & 18 & $1.19 \cdot 10^{-10}$ & 2.4143 \\
NewM & 9 & 18 & $8.83 \cdot 10^{-11}$ & 2.4143 \\
\hline
\end{tabular}

Columns "it" and "nFe" show the number of iteration and the number of function evaluations, respectively, that each method requires to satisfy the stopping criterion $\left|f\left(x_{n}\right)\right|<$ $10^{-100}$. Column $\left|f\left(x_{i}\right)\right|$ shows the absolute values of the tested function on the same level of iterative process according to the number of function evaluations. Namely, that values are given after 12 function evaluations for each method, which means that $\left|f\left(x_{i}\right)\right|$ is given after the sixth iteration for "NM", "McDWM" and "NewM", and after the fourth iteration for other methods. The last column denoted by " $r_{c}$ " displays the values that approximate theoretically determined convergence order of methods. The formula for $r_{c}$ suggested in [4] is given by

$$
r_{c}=\frac{\log \left|f\left(x_{n}\right) / f\left(x_{n-1}\right)\right|}{\log \left|f\left(x_{n-1}\right) / f\left(x_{n-2}\right)\right|} .
$$


Table 3 Numerical results for $f_{3}(x)$

\begin{tabular}{lllll}
\hline method & it & $\mathrm{nFe}$ & $\left|f\left(x_{i}\right)\right|$ & $r_{c}$ \\
\hline NM & 11 & 22 & $1.58 \cdot 10^{-4}$ & 2.0000 \\
WFM & 7 & 21 & $1.86 \cdot 10^{-4}$ & 3.0000 \\
OM & 7 & 21 & $1.83 \cdot 10^{-9}$ & 3.0000 \\
FSM & 7 & 21 & $2.47 \cdot 10^{-6}$ & 3.0000 \\
KLWM & 7 & 21 & $2.74 \cdot 10^{-7}$ & 3.0000 \\
WM & 7 & 21 & $1.53 \cdot 10^{-5}$ & 3.0000 \\
McDWM & 9 & 18 & $2.95 \cdot 10^{-9}$ & 2.4144 \\
NewM & 9 & 18 & $2.85 \cdot 10^{-9}$ & 2.4144 \\
\hline \multicolumn{5}{r}{} \\
& Table 4 Numerical results for $f_{4}(x)$ & \\
\hline method & it & $\mathrm{nFe}$ & $\left|f\left(x_{i}\right)\right|$ & $r_{c}$ \\
\hline NM & 7 & 14 & $7.03 \cdot 10^{-68}$ & 2.0000 \\
WFM & 4 & 12 & $1.22 \cdot 10^{-116}$ & 3.0000 \\
OM & 5 & 15 & $3.66 \cdot 10^{-88}$ & 3.0000 \\
FSM & 5 & 15 & $4.74 \cdot 10^{-80}$ & 3.0000 \\
KLWM & 5 & 15 & $3.39 \cdot 10^{-53}$ & 3.0000 \\
WM & 5 & 15 & $3.36 \cdot 10^{-86}$ & 3.0000 \\
McDWM & 6 & 12 & $2.00 \cdot 10^{-169}$ & 2.4139 \\
NewM & 6 & 12 & $2.73 \cdot 10^{-168}$ & 2.4129 \\
\hline
\end{tabular}

Obviously, methods McMDW and NewM have similar numerical behavior. For both methods the coefficients $r_{c}$ are very close to the theoretically obtained convergence order $1+\sqrt{2}$. In comparison to the third order methods, McMDW and NewM require more iterations, but less function evaluations to satisfy stopping criterion. Therefore, for the same number function evaluations, those two methods have significantly better results in $\left|f\left(x_{i}\right)\right|$ columns.

According to the all presented results, one can conclude that the new variant of the McDougall-Wotherspoon method preserves good theoretic and numeric properties of the original method.

\section{Acknowledgment}

The work has been supported by the Serbian Ministry of Science, Grant nos. III044006.

\section{References}

[1] C. Chun, M.Y. LEE, A new optimal eighth-order family of iterative methods for the solution of nonlinear equations, Applied Mathematics and Computation, 223 (2013), 506-519

[2] M. Frontini, E. Sormani, Some variant of Newton's method with third-order convergence, Applied Mathematics and Computation, 140 (2003), 419-426 
A variant of McDougall-Wotherspoon method for finding simple roots of nonlinear equations 61

[3] D.R. Jackett, T. McDougall, R. Feistel, D.G. Wright, S.M. GRiffies, Algorithms for density, potential temperature, conservative temperature, and the freezing temperature of seawater, Journal of Atmospheric and Oceanic Technology, 23 (2006), 1709-1728

[4] L.O. JAY, A note on Q-order of convergence, BIT Numerical Mathematics, 41 (2001), 422-429

[5] J. Kou, Y. LI, X. WANG, A modification of Newton method with third-order convergence, Applied Mathematics and Computation 181 (2006), 1106-1111

[6] T.J. McDougall, D.R. Jackett, D.G. Wright, R. Feistel, Accurate and computationally efficient algorithms for potential temperature and density of seawater, Journal of Atmospheric and Oceanic Technology, 20 (2003), 730-741

[7] T.J. MCDougall, S. Wotherspoon, A simple modification of Newton's method to achieve convergence of order $1+\sqrt{2}$, Applied Mathematics Letters, 29 (2014), 20-25

[8] A.M. Ostrows KI, Solution of equations and systems of equations, Academic Press New York, 1960

[9] A. Y. ÖZBAn, Some new variants of Newton's method, Applied Mathematics Letters, 13 (2004), 677-682

[10] P. WANG, A third-order family of Newton-like iteration methods for solving nonlinear equations, Journal of Numerical Mathematics and Stochastics, 3 (2011), 13-19

[11] S. WeERAKoon, T.G.I. Fernando, A variant of Newton's method with accelerated thirdorder convergence, Applied Mathematics Letters, 13 (2000), 87-93 